\title{
Three dimensional contact metric manifolds with Cotton solitons
}

\author{
Xiaomin CHEN
}

(Received July 11, 2020)

(Revised April 7, 2021)

\begin{abstract}
In this article we study a three dimensional contact metric manifold $M^{3}$ with Cotton solitons. We mainly consider two classes of contact metric manifolds admitting Cotton solitons. Firstly, we study a contact metric manifold with $Q \xi=\rho \xi$, where $\rho$ is a smooth function on $M$ constant along Reeb vector field $\xi$ and prove that it is Sasakian or has constant sectional curvature 0 or 1 if the potential vector field of Cotton soliton is collinear with $\xi$ or is a gradient vector field. Moreover, if $\rho$ is constant we prove that such a contact metric manifold is Sasakian, flat or locally isometric to one of the following Lie groups: $S U(2)$ or $S O(3)$ if it admits a Cotton soliton with the potential vector field being orthogonal to Reeb vector field $\xi$. Secondly, it is proved that a $(\kappa, \mu, v)$-contact metric manifold admitting a Cotton soliton with the potential vector field being Reeb vector field is Sasakian. Furthermore, if the potential vector field is a gradient vector field, we prove that $M$ is Sasakian, flat, a contact metric $(0,-4)$-space or a contact metric $(\kappa, 0)$-space with $\kappa<1$ and $\kappa \neq 0$. For the potential vector field being orthogonal to $\xi$, if $v$ is constant we prove that $M$ is either Sasakian, or a $(\kappa, \mu)$-contact metric space.
\end{abstract}

\section{Introduction}

A Cotton soliton is a metric defined on a three dimensional smooth manifold $M$ such that the following equation

$$
\mathscr{L}_{V} g+C-\sigma g=0
$$

holds for a constant $\sigma$ and one vector field $V$, called potential vector field, where $C$ is the $(0,2)$-Cotton tensor defined by

$$
C_{i j}=\frac{1}{2 \sqrt{g}} C_{n m i} \varepsilon^{n m \ell} g_{\ell j}
$$

The author is supported by Beijing Natural Science Foundation (Grant No. 1194025) and supported partially by Science Foundation of China University of Petroleum-Beijing (No. 2462020XKJS02, No. 2462020YXZZ004).

2010 Mathematics Subject Classification. Primary 53C21; Secondary 53C15.

Key words and phrases. Cotton soliton, contact metric manifold, contact metric $(\kappa, \mu, v)$-manifold, Sasakian manifold. 
in a local frame of $M$. Here $\varepsilon$ is a tensor density, in an orthonormal frame $\varepsilon^{123}=1$ and $C_{i j k}$ is Cotton tensor. As the Ricci soliton being fixed point of Ricci flow, Cotton solitons are fixed points of the Cotton flow up to diffeomorphisms and rescaling:

$$
\frac{\partial}{\partial t} g(t)=C_{g(t)}
$$

introduced in [14], where $C_{g(t)}$ is the $(0,2)$-Cotton tensor of $(M, g(t))$. Cotton soliton is said to be trivial if $C=0$ (i.e. locally conformally flat). Using the terminology of Ricci solitons, we call a Cotton soliton shrinking, steady and expanding according as $\sigma$ is positive, zero and negative respectively. If the potential vector field $V$ is a gradient field for some function, then $g$ is called a gradient Cotton soliton, i.e. the following equation

$$
2 \text { Hess } f+C=\sigma g
$$

is satisfied for a smooth function $f$ on $M$.

For a Riemannian case, in [18] it proved that a compact Riemannian Cotton soliton is locally conformally flat, and in the noncompact case the existence of a nontrivial shrinking Cotton soliton on Heisenberg group $\mathscr{H}$ is given. Meanwhile, for a non-Riemannian case, they gave the existence of Lorentzian Cotton solitons. Furthermore, E. Calviño-Louzao et al. studied left-invariant Cotton solitons on homogeneous manifolds, see [17].

In fact, Cotton solitons are closely related to Ricci and Yamabe solitons, which are defined respectively by

$$
\mathscr{L}_{V} g+R i c=\sigma g \quad \text { and } \quad \mathscr{L}_{V} g=(r-\sigma) g,
$$

where Ric and $r$ are denoted by the Ricci tensor and scalar curvature, respectively (see the examples $[16,7]$ ). We notice that many authors studied Ricci solitons and Yamabe solitons on contact metric manifolds, for instance, Cho and Sharma in $[5,6]$ studied a contact metric manifold with a Ricci soliton such that potential vector field $V$ being collinear with $\xi$, and Venkatesha-Naik [21] proved that a contact metric manifold with a Yamabe soliton is flat or it has constant scalar curvature under the assumption that $\phi Q=Q \phi$. More results can refer to $[10,11,19,20]$.

The previous works motivate us to study Cotton solitons on a three dimensional contact metric manifold. In this article, we study two classes of contact metric 3-manifolds admitting a Cotton soliton including a contact metric 3-manifolds with $Q \xi=\rho \xi$ and a $(\kappa, \mu, v)$-contact metric 3-manifold. In Section 3, for a contact metric 3-manifolds with $Q \xi=\rho \xi$, we first assume that the function $\rho$ is constant along Reeb vector field $\xi$. Such a class of contact metric manifolds was studied in [2] under the hypothesis of pseudosymmetric. 
We classify such a class of contact metric manifold admitting a Cotton soliton with potential vector field $V$ being collinear with $\xi$ or a gradient vector field. For $V$ being orthogonal to Reeb vector field, we need to assume that $\rho$ is a constant function. For a $(\kappa, \mu, v)$-contact metric manifold, in Section 4 we also consider the potential vector field of a Cotton soliton being Reeb vector field, a gradient vector field and orthogonal to $\xi$, respectively. In order to state and prove our conclusions, we need to give some preliminaries of contact manifolds, which are presented in Section 2.

\section{Preliminaries}

A contact metric manifold is a smooth manifold $M^{2 n+1}$ with a global one form $\eta$ such that $\eta \wedge(d \eta)^{n} \neq 0$ everywhere. The one form $\eta$ induces an almost contact structure $(\phi, \xi, \eta)$ on $M$, which satisfies

$$
\phi^{2}=-I+\eta \otimes \xi, \quad \eta \circ \phi=0, \quad \phi \circ \xi=0 .
$$

Here $\xi$ is a unique vector field (called Reeb or characteristic vector field) dual to $\eta$ and satisfying $d \eta(\xi, X)=0$ for all $X$. It is well-known that there exists a Riemannian metric $g$ such that

$$
d \eta(X, Y)=g(X, \phi Y), \quad g(X, \xi)=\eta(X)
$$

for any $X, Y \in \mathfrak{X}(M)$. We refer to $\left(M^{2 n+1}, \phi, \xi, \eta, g\right)$ as a contact metric manifold. A contact metric manifold $\left(M^{2 n+1}, \phi, \xi, \eta, g\right)$ for which Reeb vector field $\xi$ is Killing, i.e. $\mathscr{L}_{\xi} g=0$, is called a $K$-contact manifold.

On a contact metric manifold, we recall a operator $h=\frac{1}{2} \mathscr{L}_{\xi} \phi$, which is a self-dual operator, and $\ell=R(\cdot, \xi) \xi$. Concerning the operators the following identities, which were given in [3], are satisfied:

$$
\left\{\begin{array}{l}
h \xi=0, \quad \phi h=-h \phi, \quad \nabla_{X} \xi=-\phi X-\phi h X, \quad g(h X, Y)=g(X, h Y), \\
\operatorname{trace}(h)=\operatorname{trace}(\phi h)=0, \quad \eta \circ h=0, \\
\operatorname{trace}(\ell)=g(Q \xi, \xi)=2 n-\operatorname{trace}\left(h^{2}\right) .
\end{array}\right.
$$

If $h=0$ then we have $\mathscr{L}_{\xi} g=0$, that means that $M^{2 n+1}$ is a $K$-contact manifold.

One can define a complex structure $J$ on $M \times \mathbb{R}$ by $J\left(X, f \frac{d}{d t}\right)=(\phi X-f \xi$, $\left.\eta(X) \frac{d}{d t}\right)$ for any $X \in \mathfrak{X}(M)$ and $f \in C^{\infty}(M \times \mathbb{R})$. A contact metric structure $(\phi, \xi, \eta, g)$ is said to be normal and $M$ is called Sasakian if the corresponding complex structure $J$ on $M \times \mathbb{R}$ is integrable. A Sasakian manifold is a $K$-contact manifold and the converse does not hold, but if $\operatorname{dim} M=3$ then a $K$-contact manifold is Sasakian.

In the following we assume that $M$ is a 3-dimensional contact metric manifold. Let $U$ be the open subset where the tensor $h \neq 0$ and $U^{\prime}$ be the 
open subset such that $h$ is identically zero. $U \cup U^{\prime}$ is open dense in $M$ because $h$ is a smooth function on $M$, thus a property that is satisfied in $U^{\prime} \cup U$ is also satisfied in $M$. For any $p \in U^{\prime} \cup U$, there exists a local orthonormal frame field $\mathscr{E}=\left\{e_{1}=e, e_{2}=\phi e, e_{3}=\xi\right\}$ such that $h e=\lambda e$ and $h \phi e=-\lambda \phi e$ on $U$, where $\lambda$ is a positive non-vanishing smooth function of $M$.

First of all, we have the following lemma:

Lemma 1 ([9]). In the open subset $U$, the Levi-Civita connection $\nabla$ is given by

$$
\begin{aligned}
& \nabla_{\xi} e=a \phi e, \quad \nabla_{\xi} \phi e=-a e, \quad \nabla_{\xi} \xi=0, \\
& \nabla_{e} \xi=-(1+\lambda) \phi e, \quad \nabla_{e} e=b \phi e, \quad \nabla_{e} \phi e=-b e+(1+\lambda) \xi, \\
& \nabla_{\phi e} \xi=(1-\lambda) e, \quad \nabla_{\phi e} \phi e=c e, \quad \nabla_{\phi e} e=-c \phi e+(\lambda-1) \xi,
\end{aligned}
$$

where $a$ is a smooth function,

$$
\begin{aligned}
b & =\frac{1}{2 \lambda}[\phi e(\lambda)+A] \quad \text { with } A=\operatorname{Ric}(e, \xi), \\
c & =\frac{1}{2 \lambda}[e(\lambda)+B] \quad \text { with } B=\operatorname{Ric}(\phi e, \xi) .
\end{aligned}
$$

The components of Ricci operator $Q$ are given by

$$
\left\{\begin{array}{l}
Q e=\left(\frac{1}{2} r-1+\lambda^{2}-2 a \lambda\right) e+Z \phi e+A \xi \\
Q \phi e=Z e+\left(\frac{1}{2} r-1+\lambda^{2}+2 a \lambda\right) \phi e+B \xi \\
Q \xi=A e+B \phi e+2\left(1-\lambda^{2}\right) \xi
\end{array}\right.
$$

where $Z=\xi(\lambda)$ and the scalar curvature

$$
r=\operatorname{trace}(Q)=2\left(1-\lambda^{2}-b^{2}-c^{2}+2 a+e(c)+\phi e(b)\right) .
$$

Moreover, it follows from Lemma 1 that

$$
\left\{\begin{array}{l}
{[e, \phi e]=\nabla_{e} \phi e-\nabla_{\phi e} e=-b e+c \phi e+2 \xi} \\
{[e, \xi]=\nabla_{e} \xi-\nabla_{\xi} e=-(a+\lambda+1) \phi e} \\
{[\phi e, \xi]=\nabla_{\phi e} \xi-\nabla_{\xi} \phi e=(a-\lambda+1) e}
\end{array}\right.
$$

Putting $X=e, Y=\phi e$ and $Z=\xi$ in the Jacobi identity $[[X, Y], Z]+[[Y, Z], X]$ $+[[Z, X], Y]=0$ and using (9), we conclude

$$
\left\{\begin{array}{l}
b(a+\lambda+1)-\xi(c)-\phi e(\lambda)-\phi e(a)=0, \\
c(a-\lambda+1)+\xi(b)+e(\lambda)-e(a)=0 .
\end{array}\right.
$$

Proposition 1. If the Reeb vector field $\xi$ is an eigenvector of $Q$, in the open subset $U$ the components of $(0,2)$-Cotton tensor $C$ can be expressed 
as follows:

$$
\begin{aligned}
& C_{11}=C(e, e)=-(1-\lambda)\left(\frac{1}{2} r-3+3 \lambda^{2}-2 a \lambda\right)-\xi(Z)+4 a^{2} \lambda, \\
& C_{12}=C(e, \phi e)=-2 \lambda \xi(a)-4 a Z-(1-\lambda) Z+\frac{1}{4} \xi(r), \\
& C_{13}=C(e, \xi)=e(Z)-4 a b \lambda-\phi e\left(\lambda^{2}-2 a \lambda\right)-2 c Z-\frac{1}{4} \phi e(r), \\
& C_{22}=C(\phi e, \phi e)=\xi(Z)-4 a^{2} \lambda-(1+\lambda)\left(\frac{1}{2} r-3+3 \lambda^{2}+2 a \lambda\right), \\
& C_{23}=C(\phi e, \xi)=e\left(\lambda^{2}+2 a \lambda\right)+2 b Z-\phi e(Z)-4 a c \lambda+\frac{1}{4} e(r), \\
& C_{33}=C(\xi, \xi)=r+4 a \lambda^{2}-6\left(1-\lambda^{2}\right) .
\end{aligned}
$$

Proof. It is well-known that the Cotton tensor is defined by

$$
C(X, Y) Z=\left(\nabla_{X} S\right)(Y, Z)-\left(\nabla_{Y} S\right)(X, Z)
$$

for all $X, Y, Z$, where

$$
S(X, Y)=\operatorname{Ric}(X, Y)-\frac{r}{4} g(X, Y)
$$

is the Schouten tensor. In the frame field $\mathscr{E}$, by $(2)$ the $(0,2)$-Cotton tensor is simplified as

$$
C_{i j}=\frac{1}{2} C_{n m i} \varepsilon^{n m j}, \quad i, j=1,2,3,
$$

where $C_{i j k}=C\left(e_{i}, e_{j}\right) e_{k}$. It is clear that $C_{i j k}=-C_{j i k}$ and $C_{i i k}=0$ for all $i, j, k$. Thus

$$
\begin{aligned}
C_{11} & =\frac{1}{2} C_{n m 1} \varepsilon^{n m 1}=\frac{1}{2} C_{1 m 1} \varepsilon^{1 m 1}+\frac{1}{2} C_{2 m 1} \varepsilon^{2 m 1}+\frac{1}{2} C_{3 m 1} \varepsilon^{3 m 1} \\
& =\frac{1}{2} C_{231} \varepsilon^{231}+\frac{1}{2} C_{321} \varepsilon^{321}=C_{231} .
\end{aligned}
$$

Analogously, we have

$$
C_{12}=C_{311}, \quad C_{13}=C_{121}, \quad C_{22}=C_{312}, \quad C_{23}=C_{122}, \quad C_{33}=C_{123} .
$$

Since $\xi$ is an eigenvector of $Q$, by the third term of (7) we have $A=B=0$. Next, making use of (17) and Lemma 1, we directly compute the components of $C$ as follows: 


$$
\begin{aligned}
& C_{11}=\left(\nabla_{e_{2}} S\right)\left(e_{3}, e_{1}\right)-\left(\nabla_{e_{3}} S\right)\left(e_{2}, e_{1}\right)=\left(\nabla_{\phi e} R i c\right)(\xi, e)-\left(\nabla_{\xi} R i c\right)(\phi e, e) \\
& =-\operatorname{Ric}\left(\nabla_{\phi e} \xi, e\right)-\operatorname{Ric}\left(\xi, \nabla_{\phi e} e\right)-\xi(Z)+\operatorname{Ric}\left(\nabla_{\xi} \phi e, e\right)+\operatorname{Ric}\left(\phi e, \nabla_{\xi} e\right) \\
& =-(1-\lambda)\left(\frac{1}{2} r-1+\lambda^{2}-2 a \lambda\right)-2(\lambda-1)\left(1-\lambda^{2}\right) \\
& -\xi(Z)-a\left(\frac{1}{2} r-1+\lambda^{2}-2 a \lambda\right)+a\left(\frac{1}{2} r-1+\lambda^{2}+2 a \lambda\right) \\
& =-(1-\lambda)\left(\frac{1}{2} r-3+3 \lambda^{2}-2 a \lambda\right)-\xi(Z)+4 a^{2} \lambda, \\
& C_{12}=\left(\nabla_{e_{3}} S\right)\left(e_{1}, e_{1}\right)-\left(\nabla_{e_{1}} S\right)\left(e_{3}, e_{1}\right)=\left(\nabla_{\xi} R i c\right)(e, e)-\left(\nabla_{e} R i c\right)(\xi, e)-\frac{1}{4} \xi(r) \\
& =\xi\left(\frac{1}{2} r-1+\lambda^{2}-2 a \lambda\right)-2 \operatorname{Ric}\left(\nabla_{\xi} e, e\right)+\operatorname{Ric}\left(\nabla_{e} \xi, e\right)+\operatorname{Ric}\left(\xi, \nabla_{e} e\right)-\frac{1}{4} \xi(r) \\
& =\xi\left(\frac{1}{2} r-1+\lambda^{2}-2 a \lambda\right)-2 a Z-(1+\lambda) Z-\frac{1}{4} \xi(r) \\
& =-2 \lambda \xi(a)-4 a Z-(1-\lambda) Z+\frac{1}{4} \xi(r), \\
& C_{13}=\left(\nabla_{e_{1}} S\right)\left(e_{2}, e_{1}\right)-\left(\nabla_{e_{2}} S\right)\left(e_{1}, e_{1}\right)=\left(\nabla_{e} R i c\right)(\phi e, e)-\left(\nabla_{\phi e} R i c\right)(e, e)+\frac{1}{4} \phi e(r) \\
& =e(Z)-\operatorname{Ric}\left(\nabla_{e} \phi e, e\right)-\operatorname{Ric}\left(\phi e, \nabla_{e} e\right)-\phi e\left(\frac{1}{2} r-1+\lambda^{2}-2 a \lambda\right) \\
& +2 \operatorname{Ric}\left(\nabla_{\phi e} e, e\right)+\frac{1}{4} \phi e(r) \\
& =e(Z)-4 a b \lambda-\phi e\left(\frac{1}{2} r-1+\lambda^{2}-2 a \lambda\right)-2 c Z+\frac{1}{4} \phi e(r) \\
& =e(Z)-4 a b \lambda-\phi e\left(\lambda^{2}-2 a \lambda\right)-2 c Z-\frac{1}{4} \phi e(r), \\
& C_{22}=\left(\nabla_{e_{3}} S\right)\left(e_{1}, e_{2}\right)-\left(\nabla_{e_{1}} S\right)\left(e_{3}, e_{2}\right)=\left(\nabla_{\xi} R i c\right)(e, \phi e)-\left(\nabla_{e} R i c\right)(\xi, \phi e) \\
& =\xi(Z)-\operatorname{Ric}\left(\nabla_{\xi} e, \phi e\right)-\operatorname{Ric}\left(e, \nabla_{\xi} \phi e\right)+\operatorname{Ric}\left(\nabla_{e} \xi, \phi e\right)+\operatorname{Ric}\left(\xi, \nabla_{e} \phi e\right) \\
& =\xi(Z)-4 a^{2} \lambda-(1+\lambda)\left(\frac{1}{2} r+2 a \lambda\right)+3(1+\lambda)\left(1-\lambda^{2}\right), \\
& C_{23}=\left(\nabla_{e_{1}} S\right)\left(e_{2}, e_{2}\right)-\left(\nabla_{e_{2}} S\right)\left(e_{1}, e_{2}\right) \\
& =\left(\nabla_{e} R i c\right)(\phi e, \phi e)-\left(\nabla_{\phi e} R i c\right)(e, \phi e)-\frac{1}{4} e(r)
\end{aligned}
$$




$$
\begin{aligned}
= & e\left(\frac{1}{2} r-1+\lambda^{2}+2 a \lambda\right)-2 \operatorname{Ric}\left(\nabla_{e} \phi e, \phi e\right)-\phi e(Z)+\operatorname{Ric}\left(\nabla_{\phi e} e, \phi e\right) \\
& +\operatorname{Ric}\left(e, \nabla_{\phi e} \phi e\right)-\frac{1}{4} e(r) \\
= & e\left(\lambda^{2}+2 a \lambda\right)+2 b Z-\phi e(Z)-4 a c \lambda+\frac{1}{4} e(r), \\
C_{33}= & \left(\nabla_{e_{1}} S\right)\left(e_{2}, e_{3}\right)-\left(\nabla_{e_{2}} S\right)\left(e_{1}, e_{3}\right)=\left(\nabla_{e} \operatorname{Ric}\right)(\phi e, \xi)-\left(\nabla_{\phi e} \operatorname{Ric}\right)(e, \xi) \\
= & -\operatorname{Ric}\left(\nabla_{e} \phi e, \xi\right)-\operatorname{Ric}\left(\phi e, \nabla_{e} \xi\right)+\operatorname{Ric}\left(\nabla_{\phi e} e, \xi\right)+\operatorname{Ric}\left(e, \nabla_{\phi e} \xi\right) \\
= & \left(\frac{1}{2} r+2 a \lambda\right)(1+\lambda)-6\left(1-\lambda^{2}\right)+(1-\lambda)\left(\frac{1}{2} r-2 a \lambda\right) \\
= & r+4 a \lambda^{2}-6\left(1-\lambda^{2}\right) .
\end{aligned}
$$

This completes the proof.

\section{Contact metric 3-manifolds with $Q \xi=\rho \xi$}

First we assume that the function $\rho$ is constant along Reeb vector field $\xi$ and prove the following conclusion.

THEOREM 1. Let $\left(M^{3}, \phi, \xi, \eta, g\right)$ be a contact metric manifold such that $Q \xi=\rho \xi$, where $\rho$ is a smooth function on $M^{3}$ constant along Reeb vector field $\xi$. If $M$ admits a Cotton soliton with potential vector field being collinear with Reeb vector field $\xi$, then $M$ either is Sasakian, or has constant sectional curvature 0 or 1 .

Proof. We can denote $U^{\prime}$ and $U$ as follows:

$$
\begin{gathered}
U^{\prime}=\{p \in M: \lambda=0 \text { in a neighborhood of } p\}, \\
U=\{p \in M: \lambda \neq 0 \text { in a neighborhood of } p\} .
\end{gathered}
$$

If $M=U^{\prime}$, then $M$ is Sasakian. In the following we assume that $U$ is not empty, and let $\{e, \phi e, \xi\}$ be a $\phi$-basis in $U$.

The assumption that $Q \xi=\rho \xi$ and (7) imply $A=B=0$ and $\rho=2\left(1-\lambda^{2}\right)$, where $\xi(\rho)=0$. From this we know $Z=\xi(\lambda)=0$.

If $V=0$ then Cotton equation (1) becomes $C=\sigma g$. Since the $(0,2)-$ tensor $C$ is trace-free, we see that $\sigma$ must vanish, thus $M$ is locally conformally flat. By Theorem 4.1 of [8], $M$ has constant sectional curvature 0 or 1 .

Next we suppose that $V=f \xi$ for some non-zero smooth function $f$. Then in view of (4), for any $X, Y \in \mathfrak{X}(M)$, Cotton soliton equation (1) may be 
expressed as

$$
-2 f g(\phi h X, Y)+X(f) \eta(Y)+Y(f) \eta(X)+C(X, Y)=\sigma g(X, Y) .
$$

Letting $X=Y=e$ in (18) and recalling (11) imply

$$
-(1-\lambda)\left(\frac{1}{2} r-2 a \lambda\right)+4 a^{2} \lambda+3(1-\lambda)\left(1-\lambda^{2}\right)=\sigma .
$$

Similarly, letting $X=Y=\phi e$ in (18) and recalling (14) give

$$
-4 a^{2} \lambda-(1+\lambda)\left(\frac{1}{2} r+2 a \lambda\right)+3(1+\lambda)\left(1-\lambda^{2}\right)=\sigma
$$

and putting $X=e$ and $Y=\phi e$ in (18) and using (12) give

$$
-2 \lambda \xi(a)+\frac{1}{4} \xi(r)=2 \lambda f .
$$

Now using (19) to plus (20) implies

$$
2 \sigma=-r-4 a \lambda^{2}+6\left(1-\lambda^{2}\right) .
$$

Comparing (22) with (19), we conclude

$$
2 a\left(2 a+1-\lambda^{2}\right)=\sigma .
$$

Moreover, differentiating this along $\xi$ implies

$$
\left(4 a+1-\lambda^{2}\right) \xi(a)=0
$$

since $\sigma$ is constant and $\xi(\lambda)=0$.

If $\xi(a)=0$ then differentiating (22) along $\xi$ yields $\xi(r)=0$. By (21), we have $f=0$ since $\lambda>0$. This shows that Cotton soliton is trivial.

If $\xi(a) \neq 0$ on some open subset $\mathcal{O} \subset U$, then $\lambda^{2}=1+4 a$ by (23). Therefore, by differentiating this along $\xi$, we see $\xi(a)=0$. This is a contradiction.

We complete the proof theorem.

For a gradient Cotton soliton on $M^{3}$, we prove the following conclusion.

THEOREM 2. Let $\left(M^{3}, \phi, \xi, \eta, g\right)$ be a contact metric manifold such that $Q \xi=\rho \xi$, where $\rho$ is a smooth function on $M$ constant along Reeb vector field $\xi$. If $M$ admits a gradient Cotton soliton, then $M$ either is Sasakian, or has constant sectional curvature 0 or 1.

Proof. As before if $M=U^{\prime}$ then $M$ is Sasakian. Let $\{e, \phi e, \xi\}$ be a $\phi$-basis in non-empty set $U$. First we write the potential vector field

$$
V=\nabla f=f_{1} e+f_{2} \phi e+f_{3} \xi
$$


where $f_{1}, f_{2}, f_{3}$ are three smooth functions on $M$. Since $C$ is divergence-free, we have $Q \nabla f=0$ (see [18, Remark 3]). Hence we derive from (7) that

$$
\begin{aligned}
& f_{1}\left(\frac{1}{2} r-1+\lambda^{2}-2 a \lambda\right)=0, \quad f_{2}\left(\frac{1}{2} r-1+\lambda^{2}+2 a \lambda\right)=0, \\
& f_{3}\left(1-\lambda^{2}\right)=0 .
\end{aligned}
$$

If $V=0$ then Cotton soliton is trivial as in the proof of Theorem 1. Now we assume that at least one of $f_{1}, f_{2}, f_{3}$ is nonzero. Next we will divide into two cases to discuss.

Case I: If $\lambda=1$ then $b=c=0$ by (5) and (6). Moreover, Equation (8) implies $r=4 a$, thus it follows from the second term of Equation (24) that $a f_{2}=0$.

For every Riemannian manifold we recall the following well-known formula:

$$
\frac{1}{2} \nabla r=\operatorname{div} Q
$$

Making use of (7) and the above formula, a direct computation deduces that $\nabla a=0$, i.e. $a$ is constant. If $a=0$ all components of $C$ are zero, that means that $M$ is locally conformally flat. If $a \neq 0$, then $f_{2}=0$. By Proposition 1, the components of $C$ become

$$
\begin{aligned}
& C_{11}=4 a^{2}, \quad C_{12}=0, \quad C_{13}=0, \\
& C_{22}=-4 a^{2}-8 a, \quad C_{23}=0, \quad C_{33}=8 a .
\end{aligned}
$$

For any $X, Y \in \mathfrak{X}(M)$, the gradient Cotton soliton equation (3) is expressed as

$$
2 g\left(\nabla_{X} \nabla f, Y\right)+C(X, Y)=\sigma g(X, Y) .
$$

By taking $X=Y=e$ in (26) and using (25), we get

$$
2 e\left(f_{1}\right)+4 a^{2}=\sigma
$$

and taking $X=\xi$ and $Y=e$ in (26) gives $\xi\left(f_{1}\right)=0$. Finally, putting $X=\phi e$ and $Y=e$ in (26) implies $\phi e\left(f_{1}\right)=0$ since $\lambda=1$. By the third term of (9) acting on $f_{1}$, we find $e\left(f_{1}\right)=0$, which shows $\sigma=4 a^{2}$. Moreover, putting $X=Y=\phi e$ in (26) gives

$$
-4 a^{2}-8 a=\sigma=4 a^{2}
$$

which shows $a=-1$. 
Similarly, we can obtain from (26) that $\phi e\left(f_{3}\right)=0, e\left(f_{3}\right)=0$ and $\xi\left(f_{3}\right)+8 a=\sigma=4 a^{2}$, i.e. $\xi\left(f_{3}\right)=12$ as $a=-1$. However, the first term of (9) acting on $f_{3}$ implies $\xi\left(f_{3}\right)=0$ because $b=c=0$. This leads to a contradiction.

Case II: If $\lambda \neq 1$ in some open set $\mathcal{O} \subset U$ then $f_{3}=0$ by the third term of (24). Putting $X=Y=\xi$ in (26) and using (16) we have

$$
r+4 a \lambda^{2}-6\left(1-\lambda^{2}\right)=\sigma .
$$

Letting $X=e$ and $Y=\xi$ in (26), we conclude from (13) and (5) that

$$
2 f_{2}(1+\lambda)-\left(4 b \lambda^{2}-2 \lambda \phi e(a)\right)-\frac{1}{4} \phi e(r)=0 .
$$

Similarly, letting $X=\phi e$ and $Y=\xi$ in (26), we conclude from (6) and (15) that

$$
2 f_{1}(\lambda-1)+4 c \lambda^{2}+2 \lambda e(a)+\frac{1}{4} e(r)=0 .
$$

Next we consider the following open sets:

$$
\begin{aligned}
& \mathcal{O}_{1}=\left\{p \in \mathcal{O}: \frac{1}{2} r-1+\lambda^{2}-2 a \lambda \neq 0 \text { in a neighborhood of } p\right\}, \\
& \mathcal{O}_{2}=\left\{p \in \mathcal{O}: \frac{1}{2} r-1+\lambda^{2}-2 a \lambda=0 \text { in a neighborhood of } p\right\},
\end{aligned}
$$

where the set $\mathcal{O}_{1} \cup \mathcal{O}_{2}$ is open and dense in the closure of $\mathcal{O}_{\text {. In the }}$ set $\mathcal{O}_{1}$, it implies $f_{1}=0$ from the first term of $(24)$. Since $f_{3}=0$, we must have that $f_{2} \neq 0$ in $\mathcal{O}_{1}$. Hence the second term of (24) yields

$$
\frac{1}{2} r-1+\lambda^{2}+2 a \lambda=0
$$

By comparing it with (27), we get

$$
-4\left(1-\lambda^{2}\right)-4 a\left(\lambda-\lambda^{2}\right)=\sigma .
$$

Since Poincare Lemma $d^{2} f=0$, i.e. the relation

$$
g\left(\nabla_{X} \nabla f, Y\right)=g\left(\nabla_{Y} \nabla f, X\right)
$$

holds for any $X, Y \in \mathfrak{X}(M)$, letting $X=\xi$ and $Y=e$ in (31) and using Lemma 1 , we obtain

$$
a=-1-\lambda
$$


Substituting this into (30) implies that $\lambda$ and $a$ are constants. Thus $b=c=0$ by (5) and (6). Furthermore, it follows from (27) that $r$ is also constant. Recalling (28), we find $f_{2}(1+\lambda)=0$. This shows $f_{2}=0$ since $\lambda>0$ in $\mathcal{O}$. The contradiction means that $\mathcal{O}_{1}$ is empty.

In $\mathrm{O}_{2}$, the following relation holds:

$$
\frac{1}{2} r-1+\lambda^{2}-2 a \lambda=0
$$

Then $a f_{2}=0$ by the second term of (24). Write

$$
\mathscr{V}_{1}=\left\{p \in \mathcal{O}_{2}: a \neq 0\right\} \quad \text { and } \quad \mathscr{V}_{2}=\left\{p \in \mathcal{O}_{2}: a=0\right\} .
$$

Here $\mathscr{V}_{1} \cup \mathscr{V}_{2}$ is the open and dense in the closure of $\mathscr{O}_{2}$. Then $f_{2}=0$ in $\mathscr{V}_{1}$. Letting $X=\xi$ and $Y=\phi e$ in (31) and using Lemma 1, we obtain

$$
a=-1+\lambda
$$

since $f_{1} \neq 0$ in $\mathscr{V}_{1}$. Adopting analogous method as before, we can prove that $b=c=0$ and $a, r$ are constants. Thus (29) implies $f_{1}=0$. The contradiction shows that $\mathscr{V}_{1}$ is empty. Thus $a=0$ in $\mathcal{O}_{2}$ and it implies from (32) that

$$
r=2\left(1-\lambda^{2}\right)
$$

Inserting this into (27) implies $\sigma=-4\left(1-\lambda^{2}\right)$. This shows that $r$ is constant and $b=c=0$. However, Equations (28) and (29) yield $f_{1}=f_{2}=0$ since $\lambda \neq 1$. It is impossible.

We complete the proof of theorem.

Furthermore, for the potential vector field $V$ being orthogonal to $\xi$, we need more strong hypothesis that $\rho$ is constant.

THEOREM 3. Let $\left(M^{3}, \phi, \xi, \eta, g\right)$ be a contact metric manifold such that $Q \xi=\rho \xi$, where $\rho$ is constant. If $M$ admits a Cotton soliton with potential vector field being orthogonal to Reeb vector field $\xi$, then $M$ is either

(a) Sasakian,

(b) flat,

(c) locally isometric to one of the following Lie groups equipped with a left invariant metric: $S U(2)$ or $S O(3)$.

Proof. Under the assumption, by the main theorem of [12], the Ricci operator is expressed as

$$
Q=\alpha I+\beta \eta \otimes \xi+\gamma h
$$


where $\alpha=\frac{1}{2}(r-2 k), \beta=\frac{1}{2}(6 k-r), \gamma=-\alpha$ and $k=\frac{1}{2}$ trace $(\ell)$. Moreover, $r$ and $\lambda=\sqrt{1-k}$ are constants. Thus we have $b=c=A=B=Z=0$ and $a=\frac{1}{2} \alpha$ is also constant from (7).

When $\lambda=0, M$ is Sasakian. In the following we assume $\lambda>0$. By Proposition 1, the components of $C$ become

$$
\left\{\begin{array}{l}
C_{11}=(1-\lambda)(\beta+\alpha \lambda)+\alpha^{2} \lambda, \\
C_{12}=0, \quad C_{13}=0, \\
C_{22}=(1+\lambda)(\beta-\alpha \lambda)-\alpha^{2} \lambda, \\
C_{23}=0, \quad C_{33}=2 \alpha+2 \alpha \lambda^{2}-4 k .
\end{array}\right.
$$

Set $V=f_{1} e+f_{2} \phi e$, where $f_{1}, f_{2}$ are smooth functions on $M$. For any $X, Y \in \mathfrak{X}(M)$, Cotton soliton equation (1) is rewritten as

$$
g\left(\nabla_{X} V, Y\right)+g\left(\nabla_{Y} V, X\right)+C(X, Y)=\sigma g(X, Y) .
$$

Putting $X=Y=e$ in (34), it follows from Lemma 1 and (33) that

$$
2 e\left(f_{1}\right)+(1-\lambda)(\beta+\alpha \lambda)+\alpha^{2} \lambda=\sigma .
$$

Putting $X=Y=\phi e$ in (34), it follows from Lemma 1 that

$$
2 \phi e\left(f_{2}\right)+(1+\lambda)(\beta-\alpha \lambda)-\alpha^{2} \lambda=\sigma .
$$

Similarly, putting $X=Y=\xi$ in (34) and using (33) we have

$$
\sigma=2 \alpha+2 \alpha \lambda^{2}-4 k
$$

Letting $X=e$ and $Y=\xi$ in (34), it implies from Lemma 1 that

$$
f_{2}(1+\lambda-a)+\xi\left(f_{1}\right)=0 .
$$

Letting $X=e$ and $Y=\phi e$ in (34) implies

$$
e\left(f_{2}\right)+\phi e\left(f_{1}\right)=0
$$

and letting $X=\phi e$ and $Y=\xi$ in (34) implies

$$
f_{1}(\lambda-1+a)+\xi\left(f_{2}\right)=0 .
$$

Now differentiating (38) along $e$ and using (39), we have

$$
-\phi e\left(f_{1}\right)(1+\lambda-a)+e\left(\xi\left(f_{1}\right)\right)=0 .
$$

Since $e\left(f_{1}\right)$ is constant by (35), applying the second term of (9) in $f_{1}$ provides

$$
e\left(\xi\left(f_{1}\right)\right)=\xi\left(e\left(f_{1}\right)\right)-(a+\lambda+1) \phi e\left(f_{1}\right)=-(a+\lambda+1) \phi e\left(f_{1}\right) .
$$


Substituting this into previous formula gives $\phi e\left(f_{1}\right)=0$, which implies $e\left(f_{2}\right)=0$ from (39).

Further, applying the first term of (9) in $f_{1}$ and $f_{2}$ respectively provides $\xi\left(f_{1}\right)=\xi\left(f_{2}\right)=0$. Therefore (38) and (40) become

$$
f_{2}(1+\lambda-a)=0 \quad \text { and } \quad f_{1}(\lambda-1+a)=0 .
$$

If $1+\lambda-a=0$ then $f_{1}=0$. Applying the second term of (9) in $f_{2}$ provides

$$
e\left(\xi\left(f_{2}\right)\right)-\xi\left(e\left(f_{2}\right)\right)=-(a+\lambda+1) \phi e\left(f_{2}\right)=-2(\lambda+1) \phi e\left(f_{2}\right) .
$$

This shows that $\phi e\left(f_{2}\right)=0$, i.e. $f_{2}$ is constant. Moreover, (35) and (36) become

$$
\begin{aligned}
& (1+\lambda)(\beta-\alpha \lambda)-\alpha^{2} \lambda=\sigma, \\
& (1-\lambda)(\beta+\alpha \lambda)+\alpha^{2} \lambda=\sigma .
\end{aligned}
$$

The above equations, combining the relation $\beta=2 k-\alpha$ and (37), imply

$$
2 \alpha-2 k+\alpha^{2}=0 \quad \text { and } \quad 2 \alpha-2 k-\alpha k=0 .
$$

That is, $\alpha=-k$. Because $1+\lambda=a=\frac{1}{2} \alpha$ and $\lambda^{2}=1-k$, we get $\alpha=8$ and $\lambda=3$. Equation (9) becomes

$$
[e, \phi e]=2 \xi, \quad[\xi, e]=8 \phi e, \quad[\phi e, \xi]=2 e .
$$

Thus $M$ is locally isometric to $S U(2)$ or $S O(3)$ according to [12, Theorem 3].

If $\lambda-1+a=0$ then $f_{2}=0$ by (41). Applying the third term of (9) in $f_{1}$ provides

$$
0=\phi e\left(\xi\left(f_{1}\right)\right)-\xi\left(\phi e\left(f_{1}\right)\right)=(a-\lambda+1) e\left(f_{1}\right)=-2(\lambda-1) e\left(f_{1}\right) .
$$

For $\lambda=1$, then $k=0$ and $a=0$. Therefore, by (35) and (37), we have $e\left(f_{1}\right)=$ $4 \alpha-\alpha^{2}=0$. In this case $M$ is flat and $f_{1}$ is constant. When $\lambda \neq 1$, Equation (42) shows that $e\left(f_{1}\right)=0$, i.e. $f_{1}$ is constant. As before, from (35), (36) and (37) we can obtain $\alpha=8$ and $\lambda=-3$. It is impossible.

Summing up the above discussion, we thus complete the proof of theorem.

4. $(\kappa, \mu, v)$-contact metric 3-manifolds

Definition 1 ([13]). A contact metric manifold $\left(M^{3}, \phi, \xi, \eta, g\right)$ is called a $(\kappa, \mu, v)$-contact metric manifold if the curvature tensor satisfies the condition 


$$
\begin{aligned}
R(X, Y) \xi= & \kappa(\eta(Y) X-\eta(X) Y)+\mu(\eta(Y) h X-\eta(X) h Y) \\
& +v(\eta(Y) \phi h X-\eta(X) \phi h Y)
\end{aligned}
$$

for any vector fields $X, Y$, where $\kappa, \mu$ and $v$ are smooth functions on $M$.

In particular, if $v=0$ and $\kappa, \mu$ are constants, $M$ is said to be a $(\kappa, \mu)$ contact metric space (cf. [4]).

Lemma 2 ([15, Lemma 4.3]). For every $p \in U$, there exists an open neighborhood $W$ of $p$ and orthonormal local vector fields $e$, $\phi e$, $\xi$, defined on $W$, such that

$$
h e=\lambda e, \quad h \phi e=-\lambda \phi e, \quad h \xi=0,
$$

where $\lambda=\sqrt{1-\kappa}$.

Lemma 3. Let $\left(M^{3}, \phi, \xi, \eta, g\right)$ be a $(\kappa, \mu, v)$-contact metric manifold. Then

$$
\xi(r)=2 \xi(\kappa)=-4(1-\kappa) v .
$$

Proof. For a $(\kappa, \mu, v)$-contact metric manifold the Ricci operator may be expressed as (see [1, Eq. (3.3)]):

$$
Q=\left(\frac{1}{2} r-\kappa\right) I+\left(-\frac{1}{2} r+3 \kappa\right) \eta \otimes \xi+\mu h+v \phi h .
$$

Taking the basis $\{e, \phi e, \xi\}$, by Lemma 2, we thus have

$$
\begin{aligned}
Q \xi & =2 \kappa \xi, \\
Q e & =\left(\frac{1}{2} r-\kappa+\lambda \mu\right) e+\lambda v \phi e, \\
Q \phi e & =\left(\frac{1}{2} r-\kappa-\lambda \mu\right) \phi e+\lambda v e .
\end{aligned}
$$

It implies from (7) that $Z=\lambda v$. Now using Lemma 1, we obtain

$$
\begin{aligned}
\left(\nabla_{\xi} Q\right) \xi= & 2 \xi(\kappa) \xi, \\
\left(\nabla_{e} Q\right) e= & \nabla_{e}(Q e)-Q \nabla_{e} e=e\left(\frac{1}{2} r-\kappa+\lambda \mu\right) e+b\left(\frac{1}{2} r-\kappa+\lambda \mu\right) \phi e \\
& +e(\lambda v) \phi e+\lambda v(-b e+(1+\lambda) \xi)-b Q \phi e \\
= & e\left(\frac{1}{2} r-\kappa+\lambda \mu\right) e+b\left(\frac{1}{2} r-\kappa+\lambda \mu\right) \phi e
\end{aligned}
$$




$$
\begin{aligned}
& +e(\lambda v) \phi e+\lambda v(-b e+(1+\lambda) \xi)-b\left(\left(\frac{1}{2} r-\kappa-\lambda \mu\right) \phi e+\lambda v e\right) \\
= & \left\{e\left(\frac{1}{2} r-\kappa+\lambda \mu\right)-2 b \lambda v\right\} e+\{2 b \lambda \mu+e(\lambda v)\} \phi e+\lambda v(1+\lambda) \xi \\
\left(\nabla_{\phi e} Q\right) \phi e= & \nabla_{\phi e}(Q \phi e)-Q \nabla_{\phi e} \phi e=\phi e\left(\frac{1}{2} r-\kappa-\lambda \mu\right) \phi e+c\left(\frac{1}{2} r-\kappa-\lambda \mu\right) e \\
& +\phi e(\lambda v) e+\lambda v(-c \phi e+(\lambda-1) \xi)-c Q e \\
= & \phi e\left(\frac{1}{2} r-\kappa-\lambda \mu\right) \phi e+c\left(\frac{1}{2} r-\kappa-\lambda \mu\right) e \\
& +\phi e(\lambda v) e+\lambda v(-c \phi e+(\lambda-1) \xi)-c\left(\left(\frac{1}{2} r-\kappa+\lambda \mu\right) e+\lambda v \phi e\right) \\
= & \{\phi e(\lambda v)-2 c \lambda \mu\} e+\left\{\phi e\left(\frac{1}{2} r-\kappa-\lambda \mu\right)-2 c \lambda v\right\} \phi e+\lambda v(\lambda-1) \xi .
\end{aligned}
$$

Since $\frac{1}{2} \nabla r=\operatorname{div} Q$, which, in the basis $\{e, \phi e, \xi\}$, is written as

$$
\frac{1}{2}\{e(r) e+\phi e(r) \phi e+\xi(r) \xi\}=\left(\nabla_{e} Q\right) e+\left(\nabla_{\phi e} Q\right) \phi e+\left(\nabla_{\xi} Q\right) \xi
$$

we conclude

$$
\frac{1}{2} \xi(r)=2 \lambda^{2} v+2 \xi(\kappa)
$$

Since $\xi(\lambda)=Z=\lambda v$ and $\lambda=\sqrt{1-\kappa}$, we get the desired conclusion.

In the following we use the above two lemmas to prove our conclusions.

THEOREM 4. Let $\left(M^{3}, \phi, \xi, \eta, g\right)$ be a $(\kappa, \mu, v)$-contact metric manifold. If $M$ admits a Cotton soliton such that the potential vector field $V$ is the Reeb vector field $\xi$, then $M$ is Sasakian.

Proof. As before if $M=U^{\prime}$ then $M$ is Sasakian. Cotton soliton equation (1), for any $X, Y \in \mathfrak{X}(M)$, is expressed as

$$
-2 g(\phi h X, Y)+C(X, Y)=\sigma g(X, Y) \text {. }
$$

The relation $Q \xi=2 \kappa \xi$ shows $A=B=0$ from the third term of (7). Furthermore, $Z=\lambda v$ and $\mu=-2 a$ by (7) and (43).

Letting $X=Y=e$ in (44) and using (11) imply

$$
-(1-\lambda)\left(\frac{1}{2} r-3+3 \lambda^{2}-2 a \lambda\right)-\xi(Z)+4 a^{2} \lambda=\sigma
$$


and letting $X=Y=\phi e$ in (44) and using (14) give

$$
\xi(Z)-4 a^{2} \lambda-(1+\lambda)\left(\frac{1}{2} r+2 a \lambda\right)+3(1+\lambda)\left(1-\lambda^{2}\right)=\sigma .
$$

The previous two formulas yield

$$
2 \sigma=-r-4 a \lambda^{2}+6\left(1-\lambda^{2}\right) .
$$

Putting $X=Y=\xi$ in (44) and using (16), we have

$$
r-2 \mu(1-\kappa)-6 \kappa=\sigma .
$$

This yields $\sigma=0$ by comparing (45) with (46). That is,

$$
r=2 \mu \lambda^{2}+6 \kappa
$$

Putting $X=e$ and $Y=\phi e$ in (44) gives

$$
\lambda \xi(\mu)+2 \mu \lambda v-(1-\lambda) \lambda v+\frac{1}{4} \xi(r)=2 \lambda .
$$

Similarly, using (13) and (15) respectively, we deduce

$$
\begin{aligned}
\lambda e(v)-\lambda(4 b \lambda+\phi e(\mu))-\frac{1}{4} \phi e(r) & =0, \\
\lambda(4 c \lambda-e(\mu))-\lambda \phi e(v)+\frac{1}{4} e(r) & =0 .
\end{aligned}
$$

Here we have used $Z=\lambda v, a=-\frac{1}{2} \mu$ and Equations (5) and (6).

Because $\xi(\lambda)=Z=\lambda v$, differentiating (47) with respect to $\xi$ gives

$$
\xi(r)=2 \xi\left(\mu \lambda^{2}\right)+6 \xi(\kappa)=2 \lambda^{2} \xi(\mu)+4 \mu \nu \lambda^{2}+6 \xi(\kappa) .
$$

By Lemma 3, we see

$$
\xi(\mu)=4 v-2 \mu v
$$

Substituting (51) into (48), we obtain

$$
v=\frac{2}{3} .
$$

For a $(\kappa, \mu, v)$-contact metric manifold, we recall the following equations (see [13, Eq. (4-18)]):

$$
\begin{aligned}
& e(\kappa)-\lambda e(\mu)-\lambda \phi e(v)=0, \\
& -\phi e(\kappa)-\lambda \phi e(\mu)+\lambda e(v)=0 .
\end{aligned}
$$


Making use of (49) and (50), we obtain from (47) that

$$
\begin{gathered}
\phi e(\mu)=-4 b-4 b \mu, \\
e(\mu)=-4 c-4 c \mu .
\end{gathered}
$$

Hence, by (52), inserting this into (53) and (54) respectively gives

$$
\begin{aligned}
& 0=\phi e(v)=-4 c \lambda+4 c+4 c \mu, \\
& 0=e(v)=-4 b \lambda-4 b-4 b \mu .
\end{aligned}
$$

Next we decompose three cases to discuss.

Case I: If $b=c=0$ then $e(\mu)=\phi e(\mu)=0$, and further $e(\kappa)=\phi e(\kappa)=0$ from (53) and (54). However, the first term of (9) acting on $\kappa$ implies $\xi(\kappa)=0$. It is a contradiction since $\xi(\kappa)=-2 \lambda^{2} v \neq 0$ by $(52)$.

Case II: If $b \neq 0$ in some open set $\mathcal{O} \subset U$ then $\lambda+1=-\mu$ by (56). Inserting this into (55) gives $c(\mu+1)=0$. For $\mu=-1$, it follows from (53) and (54) that $e(\kappa)=\phi e(\kappa)=0$. It is impossible as before. Thus $c=0$, i.e. $e(\mu)=0$ in $\mathcal{O}$. Using the second term of (9) and (51), we have

$$
0=e(\xi(\mu))-\xi(e(\mu))=[e, \xi] \mu=-(a+\lambda+1) \phi e(\mu),
$$

which yields $a+\lambda+1=0$, i.e. $\lambda+1=\frac{1}{2} \mu$ since if $\phi e(\mu)=0$ it will lead to a contradiction as Case I. Recalling the previous relation $\lambda+1=-\mu$, we derive that $\mu=0$. That means that $\lambda=-1$. It is impossible.

Case III: If $c \neq 0$ in some open subset of $U$ then $\lambda-1=\mu$ by (55). Inserting this into (56) gives $b(\mu+1)=0$. In the same way as Case II, we can prove that it is impossible.

Hence we complete the proof.

TheOREM 5. Let $\left(M^{3}, \phi, \xi, \eta, g\right)$ be a $(\kappa, \mu, v)$-contact metric manifold. If $M$ admits a nontrivial gradient Cotton soliton, then one of the following statements holds:

(a) for $\kappa=1, M$ is Sasakian,

(b) for $\kappa=0, M$ is either flat or $(0,-4)$-contact metric space. In the second case $M$ is locally isometric to one of the following Lie groups: $S U(2)$ or $S O(3)$,

(c) for $\kappa<1$ and $\kappa \neq 0, M$ is a contact metric $(\kappa, 0)$-space. In this case, $M$ is locally isometric to one of the following Lie groups equipped with a left invariant metric: $S U(2)$ if $0<\kappa<1, S L(2, \mathbb{R})$ if $\kappa<0$.

Proof. If $M=U^{\prime}$ then a $(\kappa, \mu, v)$-contact metric manifold is Sasakian with $\kappa=1, \mu \in \mathbb{R}$ and $h=0$. Next we assume that $U$ is not empty and $\{e, \phi e, \xi\}$ is a $\phi$-basis as before. 
Write the potential vector field

$$
V=\nabla f=f_{1} e+f_{2} \phi e+f_{3} \xi
$$

where $f_{1}, f_{2}, f_{3}$ are three smooth functions on $M$. For any $X, Y \in \mathfrak{X}(M)$, the gradient Cotton soliton equation (3) is written as Equation (26). Since $Q \nabla f=0$, we have

$$
\begin{aligned}
& f_{1}\left(\frac{1}{2} r-\kappa+\lambda \mu\right)+f_{2} \lambda v=0, \quad f_{2}\left(\frac{1}{2} r-\kappa-\lambda \mu\right)+f_{1} \lambda v=0, \\
& f_{3} \kappa=0 .
\end{aligned}
$$

If $\kappa \equiv 0$ in $U$ then $\lambda=\sqrt{1-\kappa}=1$. We get $Z=\xi(\lambda)=\lambda v=0$, equivalently, $v=0$. Further it is easy to see that $r=4 a$ and $\mu=-2 a$ are constants. From (57), $a f_{2}=0$. If $a=0$, i.e. $\mu=0$ and in this case $M$ is flat. If $a \neq 0$ then $f_{2}=0$. Putting $X=Y=\xi$ in (26) we have

$$
2 \xi\left(f_{3}\right)+8 a=\sigma
$$

Letting $X=e$ and $Y=\xi$ in (26) implies $e\left(f_{3}\right)=0$. Moreover, letting $X=\phi e$ and $Y=\xi$ in (26) implies $\phi e\left(f_{3}\right)=0$. Because $b=c=0$, applying the first term of (9) on $f_{3}$ gives $\xi\left(f_{3}\right)=0$. Thus (58) implies $\sigma=8 a$.

On the other hand, since $g\left(\nabla_{\xi} \nabla f, \phi e\right)=g\left(\nabla_{\phi e} \nabla f, \xi\right)$, we obtain $a f_{1}=\phi e\left(f_{3}\right)$ $=0$, i.e. $f_{1}=0$. Letting $X=Y=e$ in (26) implies $2 e\left(f_{1}\right)+4 a^{2}=\sigma$, i.e. $\sigma=4 a^{2}$. Therefore we find $a=2$, i.e. $\mu=-4$. According to [4, Theorem 3], $M$ is locally isometric to one of the following Lie groups: $S U(2)$ or $S O(3)$. by

In the following we consider the case where $\kappa<1$ and $\kappa \neq 0$. Denote

$$
U_{1}=\{p \in U: \kappa(p) \neq 0 \text { and } \kappa(p)<1\} .
$$

Then $f_{3}=0$ in $U_{1}$. Putting $X=Y=\xi$ in (26) we have

$$
r-2 \mu(1-\kappa)-6 \kappa=\sigma .
$$

Since $\sigma$ is constant, differentiating (59) along $\xi$ and using Lemma 2, we also obtain Equation (51).

Because at least one of $f_{1}$ and $f_{2}$ is nonzero, the first and second terms of (57) imply

$$
(1-\kappa)\left(\mu^{2}+v^{2}\right)=\left(\frac{1}{2} r-\kappa\right)^{2}
$$


Next we prove $v \equiv 0$ in $U_{1}$. Since $Z=\lambda v$ and $a=-\frac{1}{2} \mu$, letting $X=e$ and $Y=\phi e$ in (26) gives

$$
2 b f_{1}+2 e\left(f_{2}\right)+\lambda \xi(\mu)+2 \mu \lambda v-(1-\lambda) \lambda v+\frac{1}{4} \xi(r)=0 .
$$

In terms of (51) and Lemma 3, the above formula becomes

$$
2 b f_{1}+2 e\left(f_{2}\right)+3 \lambda v=0 .
$$

Letting $X=e$ and $Y=\xi$ in (26) implies

$$
2 f_{2}(1+\lambda)-8 \lambda^{2} b=\frac{1}{4} \phi e(r) .
$$

Moreover, letting $X=\phi e$ and $Y=\xi$ in (26) implies

$$
2 f_{1}(\lambda-1)+8 c \lambda^{2}=-\frac{1}{4} e(r) \text {. }
$$

Here we have used Equations (53) and (54).

Using (62) and (63), we conclude from the second term of (9) that

$$
\begin{aligned}
-b e(r)+c \phi e(r)+2 \xi(r)= & {[e, \phi e] r=e(\phi e(r))-\phi e(e(r)) } \\
= & 8 e\left(f_{2}\right)(1+\lambda)+16 f_{2} c \lambda-32 \lambda^{2} e(b) \\
& +8 \phi e\left(f_{1}\right)(\lambda-1)+16 f_{1} b \lambda+32 \lambda^{2} \phi e(c) .
\end{aligned}
$$

It follows from Lemma 3 that

$$
-\lambda^{2} v=\left[e\left(f_{2}\right)+b f_{1}\right](1+\lambda)+\left[\phi e\left(f_{1}\right)+c f_{2}\right](\lambda-1)-4 \lambda^{2} e(b)+4 \lambda^{2} \phi e(c) .
$$

Since $g\left(\nabla_{\phi e} \nabla f, e\right)=g\left(\nabla_{e} \nabla f, \phi e\right)$, using Lemma 1 we see that

$$
\phi e\left(f_{1}\right)+c f_{2}=e\left(f_{2}\right)+b f_{1},
$$

thus recalling (61) we obtain from (64) that

$$
v=-2 e(b)+2 \phi e(c) .
$$

Since $A=B=0$, it follows from (5) and (6) that

$$
\begin{gathered}
e(b)=e\left(\frac{\phi e(\lambda)}{2 \lambda}\right)=\frac{e(\phi e(\lambda)) \lambda-\phi e(\lambda) e(\lambda)}{2 \lambda^{2}}, \\
\phi e(c)=\phi e\left(\frac{e(\lambda)}{2 \lambda}\right)=\frac{\phi e(e(\lambda)) \lambda-e(\lambda) \phi e(\lambda)}{2 \lambda^{2}} .
\end{gathered}
$$


Hence using the first term of (9) we have

$$
\phi e(c)-e(b)=\frac{[\phi e, e](\lambda)}{2 \lambda}=\frac{b e(\lambda)-c \phi e(\lambda)-2 \xi(\lambda)}{2 \lambda}=-v .
$$

Substituting this into (66), we find that $v=0$ on $U_{1}$. This shows $\xi(\kappa)=$ $\xi(\mu)=0$ from (51). Moreover, by (60) we know that either $\frac{1}{2} r-\kappa=\lambda \mu$ or $\frac{1}{2} r-\kappa=-\lambda \mu$.

If $\frac{1}{2} r-\kappa=\lambda \mu$ then Equation (57) implies $f_{1} \mu=0$. Consider

$$
\mathscr{V}_{1}=\left\{p \in U_{1}: f_{1}(p)=0\right\} \quad \text { and } \quad \mathscr{V}_{2}=\left\{p \in U_{1}: f_{1}(p) \neq 0\right\} .
$$

Thus $\mathscr{V}_{1} \cup \mathscr{V}_{2}$ is dense in the closure of $U_{1}$. In $\mathscr{V}_{1}$, we have $f_{2} \neq 0$. Then (61) yields $e\left(f_{2}\right)=0$, which further implies $c=0$ from (65). Recalling (6) we get $e(\lambda)=0$.

Now by using the second term of (9) on $\lambda$ we obtain $(a+\lambda+1) \phi e(\lambda)=$ 0 . If $\phi e(\lambda) \neq 0$ in some open set $\mathscr{V}_{1}^{\prime} \subset \mathscr{V}_{1}$ then $a=-\lambda-1$, i.e. $\frac{1}{2} \mu=\lambda+1$. Recalling $\kappa=1-\lambda^{2}$, we derive from (59) that

$$
4 \lambda(\lambda+1)-4 \lambda^{3}-4=\sigma .
$$

This shows that $\lambda$ is constant since $\sigma$ is constant. Consequently, $\phi e(\lambda)=0$ in $\mathscr{V}_{1}^{\prime}$. The contradiction gives $\phi e(\lambda)=0$ in $\mathscr{V}_{1}$. Namely, $\lambda$ is constant, hence it is easy to see that $r$ is constant and $b=0$. However, Equation (62) yields $\lambda=-1$, which is impossible since $f_{2} \neq 0$ and $\lambda$ is positive. This shows that $\mathscr{V}_{1}$ is empty and $\mu=0$ in $U_{1}$. We conclude from (53) and (54) that $\kappa$ is constant.

For $\frac{1}{2} r-\kappa=-\lambda \mu$, we have $\mu f_{2}=0$ from (57). In the same way, we can prove that $\mu=0$ and $\kappa$ is constant.

Summing up the above discussion, we complete the proof.

Since the condition that $v$ is constant does not imply that the other functions $\kappa$ and $v$ are constants (see [13, Remark 5.3]), we consider the case where $v$ is constant.

THEOREM 6. Let $\left(M^{3}, \phi, \xi, \eta, g\right)$ be a contact metric $(\kappa, \mu, v)$-manifold such that $v$ is constant. If $M$ admits a Cotton soliton with potential vector field $V$ being orthogonal to Reeb vector field $\xi$, then $M$ is either

(a) Sasakian,

(b) a contact metric $(\kappa, \mu)$-space. Moreover, by Theorem 3, in this case $M$ is either flat, or locally isometric to one of the following Lie groups equipped with a left invariant metric: $S U(2)$ or $S O(3)$.

Proof. We know that $Q \xi=2 \kappa \xi$ implies $A=B=0$, and $Z=\lambda v, \mu=$ $-2 a$. Then $\xi(Z)=\lambda^{2} v, e(Z)=2 \lambda c v$ and $\phi e(Z)=2 b \lambda v$. 
As before, we may set $V=f_{1} e+f_{2} \phi e$. Using Cotton soliton equation (1) we derive from Lemma 1 and Proposition 1 the following equations:

$$
\left\{\begin{array}{l}
b f_{1}+e\left(f_{2}\right)+\phi e\left(f_{1}\right)+f_{2} c+3 \lambda v=0 \\
f_{2}(1+\lambda-a)+\xi\left(f_{1}\right)-8 b \lambda^{2}=\frac{1}{4} \phi e(r) \\
f_{1}(\lambda-1+a)+\xi\left(f_{2}\right)+8 c \lambda^{2}=-\frac{1}{4} e(r) \\
2 e\left(f_{1}\right)-2 b f_{2}-(1-\lambda)\left(\frac{1}{2} r-3+3 \lambda^{2}+\mu \lambda\right)+4 a^{2} \lambda=\sigma \\
2 \phi e\left(f_{2}\right)-2 c f_{1}-4 a^{2} \lambda-(1+\lambda)\left(\frac{1}{2} r-3+3 \lambda^{2}-\mu \lambda\right)=\sigma \\
r-2 \mu(1-\kappa)-6 \kappa=\sigma
\end{array}\right.
$$

Here the first equation has used (51) and Lemma 3 and the second and third equations have used Equations (53) and (54).

Moreover, differentiating the last equation of (67) along $\xi$, we can also obtain (51). Since $v$ is constant, by (53) and (54), we have

$$
e(\mu)=\frac{e(\kappa)}{\lambda}=\frac{e\left(1-\lambda^{2}\right)}{\lambda}=-4 c \lambda
$$

and

$$
\phi e(\mu)=-\frac{\phi e(\kappa)}{\lambda}=-\frac{\phi e\left(1-\lambda^{2}\right)}{\lambda}=4 b \lambda .
$$

Here we have used (5) and (6). Using (51) and the second term of (9), we get

$$
\begin{aligned}
-(a+\lambda+1) \phi e(\mu) & =[e, \xi] \mu=e(\xi(\mu))-\xi(e(\mu)) \\
& =-2 e(\mu) v+4 \xi(c) \lambda+4 c \lambda v .
\end{aligned}
$$

Since $\mu=-2 a$ and (69) imply $\phi e(\lambda)=-\phi e(a)$, we derive from the first term of (10) that $\xi(c)=b(a+\lambda+1)$. Hence inserting (68) and (69) into the previous relation gives

$$
3 c v=-2 b(a+\lambda+1) .
$$

Using the similar method with above, we can obtain

$$
3 b v=2 c(a-\lambda+1) \text {. }
$$

Next we consider four open subsets

$$
\begin{aligned}
& U_{1}=\{p \in U: b(p) \neq 0, c(p) \neq 0\}, \\
& U_{2}=\{p \in U: b(p)=0, c(p) \neq 0\},
\end{aligned}
$$




$$
\begin{aligned}
& U_{3}=\{p \in U: b(p) \neq 0, c(p)=0\}, \\
& U_{4}=\{p \in U: b(p)=0, c(p)=0\}
\end{aligned}
$$

of $U$. Clearly, $U_{1} \cup U_{2} \cup U_{3} \cup U_{4}$ is dense in the closure of $U$.

Case I. For $p \in U$, if $p \in U_{1}$ then the previous two formulas (70) and (71) yield

$$
9 v^{2}=-4(a+\lambda+1)(a-\lambda+1) .
$$

Differentiating this along $e$ gives $a-\lambda+1=0$ since $e(a)=e(\lambda) \neq 0$ obtained from (6) and (68). On the other hand, differentiating (72) along $\phi e$ gives $a+\lambda+1=0$ since $\phi e(a)=-\phi e(\lambda) \neq 0$. Thus we obtain $\lambda=0$, which is impossible.

Case II. If $p \in U_{2}$, we have $v=0$ and $a-\lambda+1=0$ from (70) and (71). Moreover, it is easy to prove that $e(\mu) \neq 0$ and $\phi e(\mu)=\phi e(\lambda)=\phi e(\kappa)=$ $\phi e(r)=0$. By $(51)$ and $v=0$, we know $\xi(\mu)=0$. Moreover, it is easy to see that $\xi(\lambda)=\xi(\kappa)=\xi(c)=0$. Recalling $\kappa=1-\lambda^{2}$, Equation (67) becomes

$$
\left\{\begin{array}{l}
e\left(f_{2}\right)+\phi e\left(f_{1}\right)+f_{2} c=0, \\
f_{2}(1+\lambda-a)+\xi\left(f_{1}\right)=0, \\
f_{1}(\lambda-1+a)+\xi\left(f_{2}\right)+8 c \lambda^{2}=-\frac{1}{4} e(r), \\
2 e\left(f_{1}\right)-\kappa \lambda \mu+\mu^{2} \lambda=\sigma+\frac{1}{2}(1-\lambda) \sigma, \\
2 \phi e\left(f_{2}\right)-2 c f_{1}-\mu^{2} \lambda+\kappa \lambda \mu=\sigma+\frac{1}{2}(1+\lambda) \sigma .
\end{array}\right.
$$

Differentiating the third term of (73) with respect to $\phi e$ implies $\phi e\left(\xi\left(f_{2}\right)\right)=$ $-\phi e\left(f_{1}\right)(\lambda-1+a)$ and differentiating the last term of $(73)$ with respect to $\xi$ gives $\xi\left(\phi e\left(f_{2}\right)\right)=c \xi\left(f_{1}\right)$. Hence applying the third term of (9) in $f_{2}$ implies

$$
0=[\phi e, \xi]\left(f_{2}\right)=\phi e\left(\xi\left(f_{2}\right)\right)-\xi\left(\phi e\left(f_{2}\right)\right)=-\phi e\left(f_{1}\right)(\lambda-1+a)-c \xi\left(f_{1}\right) .
$$

Recalling the first and second terms of (73) we obtain

$$
e\left(f_{2}\right)(\lambda-1)+c f_{2} \lambda=0 .
$$

On the other hand, differentiating the second term of (73) along $e$ gives $e\left(\xi\left(f_{1}\right)\right)=-(\lambda+1-a) e\left(f_{2}\right)-(2 c \lambda-e(a)) f_{2}$ and differentiating the fourth term of (73) along $\xi$ gives $\xi\left(e\left(f_{1}\right)\right)=0$. Hence applying the second term of (9) in $f_{1}$ implies

$$
\begin{aligned}
-(a+\lambda+1) \phi e\left(f_{1}\right) & =[e, \xi]\left(f_{1}\right)=e\left(\xi\left(f_{1}\right)\right)-\xi\left(e\left(f_{1}\right)\right) \\
& =-(\lambda+1-a) e\left(f_{2}\right)-(2 c \lambda-e(a)) f_{2} .
\end{aligned}
$$

Recalling the first term of (73) we get

$$
(\lambda+1) e\left(f_{2}\right)=-\lambda c f_{2} .
$$


By comparing (74) with (75), we find $2 c \lambda f_{2}=0$, which shows $f_{2}=0$ since $\lambda>0$. Thus Equation (73) is simplified as

$$
\left\{\begin{array}{l}
2 f_{1} a=2 c \lambda^{2}(a-\mu)=6 a c \lambda^{2} \\
2 e\left(f_{1}\right)-\kappa \lambda \mu+\mu^{2} \lambda=\frac{1}{2}(3-\lambda) \sigma \\
-2 c f_{1}-\mu^{2} \lambda+\kappa \lambda \mu=\frac{1}{2}(3+\lambda) \sigma \\
r-2 \mu(1-\kappa)-6 \kappa=\sigma
\end{array}\right.
$$

Here we have used

$$
e(r)=e\left(2 \mu \lambda^{2}+6 \kappa\right)=-8 c \lambda^{2}(\lambda-\mu+3) .
$$

We know $a \neq 0$ in $U_{2}$, otherwise, if $a=0$ then $\lambda=1$ which implies $c=0$ from (6). By the first term of (76), we obtain $f_{1}=3 c \lambda^{2}$. Inserting this into the third term of (76) gives

$$
-6 c^{2} \lambda^{2}-\mu^{2} \lambda+\kappa \lambda \mu=\frac{1}{2}(3+\lambda) \sigma .
$$

Differentiating $f_{1}=3 c \lambda^{2}$, we have

$$
e\left(f_{1}\right)=3 \lambda^{2} e(c)+12 c^{2} \lambda^{2} .
$$

Substituting this into the second term of (76), we conclude

$$
6 \lambda^{2} e(c)+24 c^{2} \lambda^{2}-\kappa \lambda \mu+\mu^{2} \lambda=\frac{1}{2}(3-\lambda) \sigma .
$$

Furthermore, since $r=2 \mu \lambda^{2}+6 \kappa+\sigma$, it follows from (8) that

$$
e(c)=\left(1+\lambda^{2}\right) \mu+2 \kappa+c^{2}+\frac{\sigma}{2} .
$$

From (77), (78) and (79), we can eliminate the function $c$. We remark that $\kappa=1-\lambda^{2}$ and $\mu=-2 a=-2(\lambda-1)$. Therefore we see that $\lambda$ must be constant since $\sigma$ is constant. It shows that $c=0$ from (6), which is contradictory with $p \in U_{2}$.

Case III. If $p \in U_{3}$ then we have $v=0$ and $a+\lambda+1=0$. Moreover, $\phi e(\mu) \neq 0$ and $e(\mu)=e(\lambda)=e(\kappa)=e(r)=0$. Also, we have $\xi(\lambda)=\xi(\kappa)=$ $\xi(c)=0$. In the same way as Case II, we can obtain from the above formulas that $f_{1}=0$. Thus Equation (67) is simplified as

$$
\left\{\begin{array}{l}
f_{2}(1+\lambda-a)-8 \lambda^{2} b=\frac{1}{4} \phi e(r) \\
-2 b f_{2}-\kappa \lambda \mu+\mu^{2} \lambda=\frac{1}{2}(3-\lambda) \sigma \\
2 \phi e\left(f_{2}\right)-\mu^{2} \lambda+\kappa \lambda \mu=\frac{1}{2}(3+\lambda) \sigma \\
r-2 \mu(1-\kappa)-6 \kappa=\sigma
\end{array}\right.
$$


As Case II, making use of (5), (8) and the above formulas, we can also prove that $\lambda$ is constant, which is contradictory with $p \in U_{3}$.

Case IV. If $p \in U_{4}$ then $e(\mu)=\phi e(\mu)=0$. Applying the first term of (9) on $\mu$, we get $\xi(\mu)=0$, which shows that $\mu$ and $a$ are constants. Moreover, it is easy to prove that $\lambda, \kappa$ are constants and $v=0$. That shows that $M$ is a contact metric $(\kappa, \mu)$-space, equivalently, $M$ satisfies $Q \xi=\rho \xi$ with $\rho=2 \kappa$ is constant.

By Theorem 3, we complete the proof.

\section{Acknowledgement}

The author would like to thank the referee for the comments.

\section{References}

[1] K. Arslan, A. Carriazo, V. Martín-Molina and C. Murathan, The curvature tensor of $(\kappa, \mu, v)$-contact metric manifolds, Monatsh Math. 177 (2015), 331-344.

[2] F. Gouli-Andreou and E. Moutafi, Three classes of pseudosymmetric contact metric 3-manifolds, Pacific J. Math. 245 (2010), No. 1, 57-77.

[3] D. E. Blair, Riemannian geometry of contact and symplectic manifolds, Progress in Mathematics 203, Birkhäuser, Boston, 2002.

[4] D. E. Blair, T. Koufogiorgos and B. J. Papantoniou, Contact metric manifolds satisfying a nullity condition, Israel J. Math. 91 (1995), No. 1-3, 189-214.

[5] J. T. Cho, Notes on contact Ricci solitons, Proc. Edinb. Math. Soc. 54 (2011), 47-53.

[6] J. T. Cho and R. Sharma, Contact geometry and Ricci solitons, Int. J. Geom. Methods Math. Phys. 7 (2010), 951-960.

[7] E. Calviño-Louzao, J. Seoane-Bascoy, M. E. Vázquez-Abal and R. Vázquez-Lorenzo, Three-dimensional homogeneous Lorentzian Yamabe solitons, Abh. Math. Semin. Univ. Hambg. 82 (2012), 193-203.

[8] G. Calvaruso, D. Perrone and L. Vanhecke, Homogeneity on three dimensional contact metric manifolds, Israel J. Math. 114 (1999), 301-321.

[9] F. Gouli-Andreou and P. J. Xenos, On 3-dimensional contact metric manifolds with $\nabla_{\xi} \tau=0$, J. Geom. 62 (1998), No. 1-2, 154-165.

[10] A. Ghosh, Ricci solitons and contact metric manifolds, Glasgow Math. J. 55 (2013), $123-130$.

[11] A. Ghosh and R. Sharma, Sasakian metric as a Ricci soliton and related results, J. Geom. Phys. 75 (2014), 1-6.

[12] T. Koufogiorgos, On a class of contact Riemannian 3-manifolds, Results Math. 27 (1995), No. 1-2, 51-62.

[13] T. Koufogiorgos, M. Markellos and V. J. Papantoniou, The harmonicity of the Reeb vector field on contact metric 3-manifolds, Pacific J. Math. 234 (2008), No. 2, 325-344.

[14] A. U. O. Kiçisel, O. Sarığlu and B. Tekin, Cotton flow, Class. Quantum Grav. 25 (2008), 165019 (15pp).

[15] T. Koufogiorgos and C. Tsichlias, On the existence of a new class of contact metric manifolds, Canad. Math. Bull. 43 (2000), No. 4, 440-447. 
[16] N. Lashkari and A. Maloney, Topologically massive gravity and Ricci-Cotton flow, Class. Quantum Grav. 28 (2011), 105007.

[17] E. Calviño-Louzao, L. M. Hervella, J. Seoane-Bascoy and R. Vázquez-Lorenzo, Homogeneous Cotton solitons, J. Phys. A: Math. Theor. 46 (2013), 285204 (19pp).

[18] E. Calviño-Louzao, E. García-Río and R. Vázquez-Lorenzo, A note on compact Cotton solitons, Class. Quantum Grav. 29 (2012), 205014 (5pp).

[19] R. Sharma, A 3-dimensional Sasakian metric as a Yamabe soliton, Inter. J. Geom. Meth. Modern Phys. 9 (2012), 1220003 (5 pages).

[20] R. Sharma, Almost Ricci solitons and $K$-contact geometry, Monatsh Math. 175 (2014), 621-628.

[21] V. Venkatesha and D. M. Naik, Yamabe solitons on 3-dimensional contact metric manifolds with $Q \phi=\phi Q$, Int. J. Geom. Methods Mod. Phys. 16 (2019), No. 03, 1950039.

\section{Xiaomin Chen}

College of Science

China University of Petroleum-Beijing

18 Fuxue Road, Changping, Beijing, 102249, China

E-mail:xmchen@cup.edu.cn 